\title{
Fall Meeting sampling
}

\begin{abstract}
Over 930 technical papers, poster presentations, and panel discussions comprised the week-long 1984 MRS Fall Meeting, spanning 16 topical symposia. The following 18 summaries were extracted from presentations at Symposia $A, B, D, F, G, J, L, M, N$, and $O$. For further information on these papers, consult the proceedings volumes to be published in Spring 1985 (for Symposium B, see the Extended Abstract volume now available from MRS).
\end{abstract}

\section{Symposium A: Energy Beam-Solid Interactions and Transient Thermal Processing}

\section{Photon-Solid Interaction (Symposium A) \\ H. Kurz, Harvard University}

The study of non-equilibrium phenomena by optical methods has contributed considerably to our understanding of the photon-solid interaction. With the advent of powerful laser sources, the interaction of intense optical coherent radiation with condensed, highly absorbing media has likewise drawn attention to practical processing technologies. The issue which has attracted considerable interest is the behavior of semiconductors under the irradiation of short $\left(1 \times 10^{-9}-10^{-13} \mathrm{~s}\right)$ intense laser pulses because of the fascinating technological prospects for the development of new electronic structures and devices.

One of the most intense debates in recent years centers on the fundamental interpretation of pulsed laser heating and melting of semiconductor surfaces. Ample evidence is presented in this paper showing that, even on a picosecond time scale, simple thermal melting takes place.

Although there is no complete microscopic model of the melting process itself, one may question in which form the absorbed laser energy resides at the time of the transition from the solid to the liquid state. Initially the laser energy is exclusively used to excite electrons in the semiconductor. The photon energy may exceed the energy gap between the electron energy in the unperturbed ground state and the lowest excited state possible in a semiconductor. Then each photon is said to create an electron-hole pair and to impart a large kinetic energy to them. Under irradiances normally encountered in laser processing with picosecond pulses, up to $10^{21}$ electronhole pairs per $\mathrm{cm}^{3}$ are created in a time of 10 picoseconds. At these high densities, the correlation between the single excited electron and the vacant bonding state (holes) is totally destroyed by rapid collisions among the electrons, among the holes, and between electrons and holes. An electronhole plasma is formed. The energy distribution of the electronic carriers in the optically generated plasma is determined by the classical Maxwell-Boltzmann distribution characterized by a plasma temperature $T_{c}$.

However, this plasma is not isolated from the ions in the material. By time-resolved optical techniques, where a second weak picosecond pulse monitors, at a certain time delay, the changes in the optical properties of the exciting pulse, the coupling between plasma and lattice can be studied. Thus, by probing the transmission at different times after the exciting pulse, the temporal build-up of vibrational energy can be observed directly. This technique is far superior to the conventional Raman-scattering technique, where the vibrational modes are detected by the frequency shift of the scattered light. The Raman technique requires the superposition of several thousand laser shots to gain a reliable signal of scattered light and suffers from a series of other experimental complexities. As a result very confusing data have been reported in the past.

The time-resolved optical probing, however, is basically a single-shot experiment, with a far better signal-to-noise ratio. By this technique it can be demonstrated conclusively that the energy is transferred to the quanta of vibrational modes (phonons) within less than one picosecond. Exactly at the laser energy at which the transition from solid to liquid occurs, the lattice temperature reaches the melting point of silicon.

By changing the wavelength of the probing laser pulse, the formation kinetics of the electron-hole plasma can be studied independently. As expected, the build-up of the ambipolar solid-state plasma occurs instantaneously during the excitation pulse. The maximum density is limited by a three-particle interaction process called Auger recombination. In this strongly density-dependent recombination process the gained energy will be trans- ferred to a third charge carrier. The same process is responsible for the decay of the plasma after the excitation, as demonstrated in this paper. All the optical data collected in a comprehensive series of picosecond time-resolved measurements are consistent with the thermal model where the laser-induced melting is attributed to the thermal destabilization of the lattice. In the critical transition regime the amplitude of the lattice vibrations exceeds a certain fraction of the equlibrium spacing.

The dominant role of thermal processes is confirmed by accompanying photoemission studies, where the maximum electron-hole temperature $T_{c}$ is determined. An upper limit of $\mathrm{T}_{\mathrm{c}} \mathrm{N} 4000 \mathrm{~K}$ means that the heating of the plasma is effectively slowed down by an efficient and rapid energy transfer to the phonons. The rapid plasma cooling observed in photoemission experiments is consistent with the strong vibrational excitation monitored in timeresolved optical experiments. On a time scale of picoseconds, there is no evidence for the widely and repeatedly published plasma model of laserinduced phase transition and no reason for the construction of alternative nonthermal models.

\section{Rapid Thermal Annealing of Silicon (Symposium A)}

\section{T. E. Seidel, AT\&T Bell Laboratories}

Annealing processes during semiconductor fabrication have traditionally used furnace operations with annealing times of the order of one hour. Recently, various radiative heating sources (e.g., tungsten-halogen lamps) have been configured to give controlled annealing times over the range 1-100 seconds. This "rapid thermal annealing" gives researchers the ability to study effects using higher-temperature, short-time combinations. Diffusion effects are limited because of the short time, while activated processes such as dislocation removal or chemical reaction-rate limited processs take place efficiently at higher temperatures. A number of applications appear possible; however, the matter of compatibility with a "technology package," i.e., the entire process, and issues such as temperature uniformity need attention.

\section{Symposium B: Laser Chemical Processing of Semiconductor Devices}

Sensitive New Method for Surface Analysis and Material Processing Diagnostics Using Lasers (Symposium B)

\section{Chris H. Becker and Keith T. Gillen, SRI International}

Microscopic contaminants or slight variations in properties or composition of semiconductor materials can ruin commercial devices. Such defects, which are difficult to detect, are also obstacles to further miniaturization and improvement of the state-of-the-art in semiconductor device manufacturing. There is a critical need, therefore, for analytical procedures of increasing sensitivity and reliability to test and evaluate semiconductor materials and structures. SRI International has recently developed a new method that promises to help fill this need. The technique uses powerful lasers for analyzing the chemical composition of microscopic samples of materials, and is particularly suited to analyzing the surfaces of materials.

Even at its current early state of development, this method, called surface analysis by laser ionization (SALI), has demonstrated the ability to detect simultaneously all elements that are present in the top atomic layer of a surface at a concentration of one part in a million. The key component of the new technique is a powerful, focused ultraviolet laser beam. The test material is placed in a vacuum chamber, where the laser beam intersects atoms and molecules that have evaporated from or been knocked off the surface by any of several means. The laser light removes an electron from those atoms and molecules, forming positively charged ions which can then be separated into the constituent elements and detected by standard mass spectrometry techniques. 
In addition to its sensitivity, the SALI method can give quantitative information on atomic compositions without the need for specially prepared reference materials for comparison. The combination of very high sensitivity, high surface specificity, and relative ease of obtaining quantitative information makes SALI a unique general analytical tool for characterizing semiconducting material.

This new method appears to be well suited to microanalysis. It has the capacity to perform chemical analysis over areas as small as one square micrometer or less and then make compositional maps of material surfaces based on the composition for each of the small spots. Such microanalysis has yet to be done, but its implementation should be straightforward.

It has been shown that the method can also be used to tell us how the composition of material changes as a function of depth. This is done by drilling a tiny hole into the material with an ion beam or another laser beam, then analyzing the chemicals that are removed.

There are many potential applications of SALI to other materials research problems besides semiconductor technology. The method has already also been used for the chemical analysis of metals and insulators such as glass and plastics. Applications to the processing of electronic materials seem particularly attractive at this time, both for monitoring processing procedures that are conducted in vacuum environment and for analyzing the ratios of major constituents and amounts for impurities in various processed materials.

\section{Symposium D: Layered Structures, Epitaxy and Interfaces}

\section{Growth and Structure of Epitaxial Layers (Symposium D) \\ D. W. Pashley, Imperial College}

An increasingly important process used in the manufacture of semiconductor electronic devices is that of epitaxial growth. This involves depositing an extremely thin layer of a semiconducting material such as silicon or gallium arsenide onto the surface of a single crystal substrate so that the layer also grows as a single crystal. The substrate can be an insulator (e.g. alumina) or another semiconducting material, or even the same semiconducting material with a different impurity (dopant) content so that its electronic properties are different. The requirements for controlling the thickness, the chemical composition, and the structure of the layer can be very stringent, because the electronic properties of the layer, and hence the behavior of the electronic device of which it is a part, are strongly influenced by these factors. It is therefore important to understand just how the layers grow and how variations in the conditions of growth affect the detailed structure of the layers.

Epitaxial growth has been studied for many years mainly with substances and materials which are not semiconductors, and many of which are not therefore used in electronic devices. Consequently much has been learned about epitaxy, and a general understanding of some of the more important features of the process has been built up over the years. The use of electron microscopy and related techniques such as electron diffraction has been particularly important in the study of the growth and structure of the layers. The results of these extensive studies are summarized and reviewed, and some of the more important ideas and areas of understanding are discussed.

The way in which the crystalline structure of the epitaxial deposit matches the crystalline structure of the substrate on which it is grown plays an important role in the control of epitaxial growth. Both the symmetry of the arrangement of the atoms in the two crystals and the natural spacings between the atoms in the crystals are important. Good quality of epitaxial growth is often associated with a good match in the symmetry and the spacings, although this is not always true. An understanding of how the deposit layer actually forms as the deposit atoms arrive on the substrate surface is vital to a proper understanding of the structure and properties of epitaxial layers, so that much work has been done on the nucleation and growth of layers, for a variety of different substrate and deposit materials. The knowledge gained from these more general studies has been of real value to the development of acceptable techniques for the processing of semiconductor layers for devices.

One of the more important features of epitaxial layers is their crystalline perfection, or the extent to which they depart from complete perfection. In general, epitaxial growth produces layers which contain crystalline defects such as those known as dislocations. These defects are introduced during the growth of the layers, and several ways in which this happens have been identified. In addition, dislocations can be formed between the layer and its substrate, and these can have harmful effects on the performance of an electronic device. Therefore there is considerable interest in methods for avoiding the formation of defects in epitaxial layers, and these also follow from the more general studies.

The paper reviews the work mentioned above and discusses some of the more recent developments in the application of epitaxy to electronic devices. These include devices which depend upon the use of extremely thin epitaxial layers, only a small number of atom layers in thickness, for which the electronic properties are significantly different from those of the equivalent bulk material.

\section{Physics in Quantum Wells (Symposium D) \\ H. L. Stormer, AT\&T Bell Laboratories}

Over the past decade Molecular Beam Epitaxy (MBE) has developed into a versatile and reliable crystal growth technique. The resulting materials have already found many applications in opto-electronics. This high-vacuum evaporation technique, mostly employed for semiconductors, allows a controlled fabrication of multiple layers of high-purity materials on an atomic scale. The growth process, being inherently two-dimensional, is ideally suited to generate two-dimensional carrier systems in which electronic transport occurs exclusively in a plane. Such two-dimensional carrier systems have astonishing physical properties.

One of the most remarkable discoveries in these structures is the integral quantized Hall effect. At low temperatures and in high magnetic fields, the Hall conduction (ratio of the current and the Hall voltage) is found to be quantized to multiples of the ratio of physical constants $\left(\mathrm{e}^{2} / \mathrm{h}\right)$ to an accuracy as high as several parts in $10^{7}$. Simultaneously, the resistivity of the material seems to vanish completely as the temperature is lowered towards $\mathrm{T}=0$. More recently, quantization and loss of resistance has also been found with quantum numbers that are rational fractions rather than integers. This fractional quantized Hall effect is presently understood as the signature of a novel quantum liquid remotely related to superfluid helium.

The ability to fabricate multiple layers of two-dimensional systems by $\mathrm{MBE}$ allows the investigation of the thermodynamic properties of such electron systems. The magnetic field dependence of the specific heat and the magnetic moment have just been detected for the first time. Studies of this kind will develop into a powerful tool and complement the traditional transport experiments.

Two-dimensional hole systems, the complement to two-dimenstional electron systems, have also been successfully implemented by MBE. Experiments on such structures show a rich spectrum of new effects. Hole systems were instrumental in demonstrating the overriding influence of the shape of the carrier-confining potential well on the motion of the carrier in the plane perpendicular to it.

With further progress in MBE, in particular with the capability to synthesize novel material combinations, one can expect to be able to tailor new structures for the study of other fundamental physical properties in two-dimensions - and they are probably full of surprises.

\section{Symposium F: Plasma Synthesis and Etching of Electronic Materials}

\section{Amorphous Polyphosphide Thin Films Prepared by Sputtering (Symposium F)}

\section{R. Schacter, Stauffer Chemical Company}

New semiconductor materials and improved processes to prepare these and other compound semiconductors for use in electronic components have been developed by Stauffer Chemical Company. The new semiconductors, called polyphosphides, are prepared as thin films, with precisely controlled electronic and material properties. When these films are deposited on the surface of another compound semiconductor, such as indium phosphide, they provide excellent "passivation" of that surface. This feature is an important step in developing a variety of higher performance electronic components.

Stauffer's process to make the polyphosphides uses a combination of existing semiconductor technology - an ionized gas in a vacuum chamberand a proprietary means of delivering high-purity phosphorus into the chamber. This approach allows efficient use of raw materials, reduces waste and effluents, and is also applicable to the manufacture of thin films of other compound semiconductors, such as indium phosphide which is used for lasers and detectors in fiber-optic telecommunications. 


\section{Dry Etching for Nanometer-Scale Fabrication (Symposium F)}

\section{Richard E. Howard, AT\&T Bell Laboratories}

Integrated circuits are now manufactured using patterning techniques capable of producing minimum feature sizes of about one micron. To put this in perspective, a typical human hair is about $50-75$ microns in diameter and bacteria are generally several microns across. These integrated circuit patterns are formed by a lithographic process similar to that used in the printing industry, but with much higher resolution. A light-sensitive plastic coating on a wafer is exposed to light in a high-resolution camera and then developed, leaving a stencil pattern on the wafer surface. This plastic stencil can be used as a mask for etching or other processing of the wafer. By replacing light with a beam of electrons, photolithography becomes electron lithography and even higher resolution can be obtained. Patterns made with conventional photolithography have a minimum size of about one micron, but those exposed by electrons can be as small as 0.01 micron, a hundred times smaller. By using a "dry etching" or "plasma etching" process, these ultrasmall stencil patterns can be etched into the wafer surface to form transistors and other devices.

The Microscience Group at AT\&T Bell Laboratories, Holmdel, New Jersey, has developed techniques using electron beam lithography and dry etching to make transistors with minimum features as small as 0.03 micron - only about 100 atoms across. These are about 30 times smaller than the finest lines in commercial integrated circuits.

Transistors with these dimensions exhibit characteristics not seen in conventional devices. For example, the movement of a single electron in this structure can be studied to give new insight into the basic mechanisms that cause noise in electronic devices. These small transistors are now being used as basic research tools to study the behavior of ultra-small devices and to help define the limits to the continued shrinking of semiconductor electronics.

\section{Plasma Process Deposits Amorphous Metal Films at Room Temper-} ature (Symposium F)

\section{A. K. Hays, Sandia National Laboratories}

A method for plasma deposition of amorphous metal alloys has been developed at Sandia National Laboratories which permits the relatively inexpensive, conformal coating of amorphous metal alloys on large, irregularly shaped objects at room temperature. Like glass, but unlike ordinary metals, amorphous metal alloys have no crystalline structure. They can be formulated to possess properties that are far superior to their crystalline counterparts, such as unusual corrosion resistance, abrasion resistance, and strength. In the past, these alloys have been produced in bulk by rapid solidification of liquid metals. This process is, however, very expensive. A rapidly solidified metal alloy will typically cost 100 times more than a conventional crystalline alloy of similar composition. Moreover, the rapidly solidified metal is obtained in the form of a powder, ribbon, or wire. Attempts to form the metal into useful shapes by heating will generally result in crystallization and loss of the special properties associated with its amorphous structure.

For applications where the engineer is only interested in utilizing the remarkable chemical and tribological properties (i.e., corrosion and abrasion resistance) of amorphous metals this problem can be circumvented by cladding standard materials, like carbon steel, with protective amorphous metal coatings. Currently, such coatings are prepared by sputtering, evaporation, electroless chemical deposition, and ion implantation. All of these techniques suffer from problems of rapidly escalating cost and technical difficulty of production as a function of the number of components in the metal alloy formulation.

The Sandia research project addresses these problems by introducing the metals and metalloids required to formulate the amorphous metal into a reaction vessel in the form of gaseous organometallics and metalloid hydrides. This mixture is then dissociated in a radiofrequency glow discharge in the presence of a hydrogen carrier gas. For the proof of the principle experiment, $\mathrm{Ni}(\mathrm{CO})_{4}$ was selected as the organometallic and $\mathrm{PH}_{3}$ as the metalloid hydride. The resulting amorphous film was silver in appearance and consisted of nickel, phosphorus, carbon, and oxygen. The film's optical and electrical properties were similar to those of an amorphous metal formulated by more conventional techniques.

The use of a reducing carrier gas is the key to the process. Films produced using argon as a carrier gas were black in appearance and were a factor of one thousand less conductive than films produced using a hydrogen carrier gas. Auger lineshape studies provided strong evidence that this peculiar behavior could be attributed to amorphous carbon inclusions in the films produced using an argon carrier gas. When hydrogen was used as a carrier gas, carbon was present as a metal carbide.

At present the program at Sandia is primarily directed toward developing corrosion-resistant coatings containing nickel, chromium, and phosphorus, but this low-temperature deposition technique is quite general and should allow the formulation of amorphous metal alloys coatings for a wide variety of applications. In addition, the long term utility of these plasma-deposited films may hinge on issues, which have yet to be addressed, such as film porosity and adhesion.

\section{Symposium G: High-Temperature Ordered Intermetallic Alloys}

Ordered Alloys as Alternatives to Superalloys for High-Temperature Applications (Symposium $G$ )

\section{J. R. Stephens, NASA Lewis Research Center}

NASA Lewis Research Center is conducting research to identify alternate materials to the commonly used nickel-base superalloys utilized in jet engines. Superalloys consist primarily of nickel with up to 12 different elements added to the nickel to produce such high-temperature properties as strength, oxidation and corrosion resistance, and long time dependability. Four of the additions in superalloys - cobalt, chromium, tantalum, and niobium-are considered strategic metals since the United States imports nearly $100 \%$ of each of these metals and they are vital to the welfare of the nation's economy. However, the United States may expect to be faced with supply shortages and price escalation for these strategic metals due to such activities as foreign cartels, political unrest, and production limitations.

The research at NASA Lewis was prompted by the cobalt shortage in 1978-1980, along with inflated prices of cobalt, tantalum, and niobium. In order to minimize the United States aerospace industry's dependence on these four metals, a program was initiated by NASA called Conservation of Strategic Aerospace Materials (COSAM). Two major thrusts of the program involved: strategic element substitution and alternate materials. Research in the first area focused on understanding the roles of the strategic metals in nickel-base superalloys and then identifying nonstrategic substitutes. This phase of the program has been successful and is now nearing completion. The second major part of the COSAM Program-alternate materials-- has focused on the equiatomic B2 aluminides of iron and nickel. The B2 aluminides have an ordered arrangement of atoms which consists of two interpenetrating cubes with aluminum atoms located on the corners of one cube and iron or nickel located on the corners of the second cube.

Interest in the equiatomic ( $50 \%$ aluminum-50\% iron or nickel) stems from a number of reasons. Of primary importance is the absence of strategic materials since iron, nickel, and aluminum are readily available to the United States. The high melting point of the aluminides offers the potential of higher operating temperatures in jet engines than can now be achieved with superalloys. This translates into higher operating efficiency and lower fuel consumption. The light weight of the aluminides will also contribute to a decrease in fuel consumption.

Other advantages include the potential of oxidation and corrosion resistance and lower cost. A major disadvantage is the lack of ductility in the aluminides at room temperature where inadvertent rough handling could lead to breakage of jet engine components.

Research on the aluminides has focused on exploiting their hightemperature potential including deformation behavior at anticipated jet engine operating temperatures, alloying to improve high-temperature strength, and oxidation testing. In addition, the lack of ductility at room temperature is being addressed via various processing techniques which offer potential to improve ductility and alloying with minor amounts of a third element to increase ductility in the aluminide materials. The research is a cooperative program involving researchers at the NASA Lewis Research Center and NASA-sponsored researchers at universities and within industry. Initial results are promising and show that although the ordered alloys behave differently than metals, strengthening mechanisms observed in metals can also be ascribed to the intermetallics. Some ductility has been achieved in both iron aluminide and nickel aluminide by special processing techniques that produce a very small grain size in the aluminides. Experimental investigations are continuing to further develop the required properties of these promising alternate methods. 


\section{Symposium J: Electronic Packaging Materials Science}

\section{Electronic Structure and Properties of Oxide Surfaces and Interfaces} (Symposium J)

\section{A. M. Stoneham, AERE Harwell}

One goal of materials science is to control properties to suit a product. By modifying surfaces, we can both improve the final product and find faster, cheaper, or more flexible processes to acheive our aim. One important factor is the understanding of surfaces and surface defects. This scientific basis points to technical opportunities and, especially because of developments in the theoretical modeling of surfaces, avoids massive trial-and-error tests by an initial theoretical pre-selection of good candidates for improved materials. This paper covers this field for ceramic surfaces: their science, their theoretical modeling, and the control of their behavior.

Three types of surfaces are discussed: the free surface of oxides, surfaces between blocks of the same crystalline ceramic oxide rotated relative to one another (grain boundaries), and surfaces between the ceramic and another material such as a metal. The aim is to understand their behavior to control mechanical properties, like adhesion and stability, and transport properties, e.g., diffusion-controlled processes.

For free surfaces, spin resonance, electron spectroscopy, and theory have been able to identify many key defects and defect reactions. These provide models for some of the catalytic processes known to occur. In all practical applications the morphology matters, i.e., the sizes of the grains making up the ceramic, and the resulting porosity and apparent surface area. Impurity additives are well known to help reduce the porosity for protective coatings and for improving mechanical properties. One new theoretical result shows that suitable additives can stabilize high surface area ceramics. This could greatly improve catalyst substrates.

For solid-solid interfaces, major progress has been made in understanding grain boundaries. Their structures have been established mainly by theoretical modeling, and these structures show how diffusion at boundaries speeds low-temperature oxidation and why electrical conduction is enhanced by boundaries. Many applications, from brazing to microcircuits, rely on metal-ceramic interfaces. Here the scientific problems remain complex and unsolved. However, useful rules are found, relating the physical properties of the ceramic oxide, the extent of wetting of the oxide by the liquid metal, and the so-called strong metal support interaction of catalysts by metal particles on the oxide.

\section{Symposium L: Potential for Very High Strength Cement-Based Materials}

\section{Development of a High-Strength Cementitious Tooling/Molding Material (Symposium L) \\ S. Wise*, J. A. Satkowski*, B. Scheetz ${ }^{* *}$, J. M. Rizer*, M. L. MacKenzie*, and D. D. Double* \\ ${ }^{*}$ CEMCOM Research Associates, **Pennsylvania State University}

DASH $47 \odot_{\text {is }}$ the commercial name given to a high-strength cement-based composite containing graded stainless steel aggregate and fine silica. It was recently developed by CEMCOM to meet a demand in the aerospace and automobile industries for a cheaper material for making tools and molds. Conventional metal molds, usually of nickel or aluminum, involve a high material cost and require complex and expensive machining processes in manufacture. In contrast, DASH 47 is cast at room temperature over a pattern, and mold shapes of complex curvature can be directly obtained with smooth and accurate replication of surface detail.

The composition of DASH 47 is based on considerations of particle size grading of the stainless steel, cement, and fine silica constituents. In addition, chemical proportioning has been used to optimize the hardening reactions that occur during the curing treatment. The result is a cement composite of minimum permeability with exceptionally high compressive and flexural strengths. The stainless steel increases both the durability of the material and its thermal conductivity, factors which are important in a molding material for plastic forming.

DASH 47 molds can be used at relatively high temperatures (sufficient for forming plastic and resin composite parts) without degradation in surface and bulk physical properties over repeated cycles. Currently, DASH 47 is being used for production molds in the aerospace industry for autoclave forming of resin-fiber composite parts and for tools stretch-forming of metal sheet. In the automobile industry, DASH 47 molds are being used for prototype production of plastic parts by injection and blow-molding techniques. A wider range of technological applications for the new cementitious molding material is being explored.

\section{Microsilica Concrete: A Technological Breakthrough Commercialized (Symposium L)}

\section{F. Radjy, Elkem Chemicals}

Concrete, a cementitious bonded substance, is probably one of the most widely used construction materials world wide (roughly $1 \mathrm{yd}^{3} / \mathrm{person} /$ year in the United States). It is, in fact, difficult to imagine today's world without concrete. The widespread use of this material is a consequence of its many attributes, including: formability; ease of adaptability to local, practically $75 \%$ earth-raw constituent ingredients; the relatively low level of sophistication needed for its field use; and, finally, its strength and durability.

Concrete has been characterized as a "forgiving material," since only with fairly imprecise control, a good quality product can be produced. The combined attributes of the "low level of needed field sophistication" together with the "forgiving nature" may be characterized as "constructibility."

Although over the years major improvements in concrete technology and products have occurred, its technological progress has been both relatively limited and unsophisticated compared to advances in other material systems. Since commercial and strategic parameters generally drive such developments, concrete's "forgiveness/unsophisticated" nature, namely constructibility, has probably been the controlling parameter, nullifying or limiting the commercial rooting of major technological potentials. In other words, market penetration of more advanced concrete products has been curtailed whenever the trade-off expense has resulted in more sophisticated, less forgiving field requirements, detracting from constructiblity.

Of the advances that have occurred, many closely correlate to developments in "additives technology." The commercial success in this area stems from the capability of improving engineering performance along with better constructibility; or, at the very least, not sacrificing constructibility. The performance improvements attained through the use of these traditional additives have been significant but still limited and expressible by percentages and not factors.

While microsilica additives build on the existing additives technology and satisfy the constructibility requirements, their effectiveness goes far beyond the standard additives. In fact, the commercial introduction of microsilica additives positions the concrete industry at a new point of departure, with a technology leap becoming feasible. Properties and microstructure can now be changed by factors to orders of magnitude, instead of by percentages. In terms of strengths, for instance, first generation microsilica additives are enabling field-producible concrete strengths of up to 17,000 psi.

Even though in North America microsilica concrete has become available only during the recent years, and worldwide high-performance applications have been evolving during the past three to five years, microsilica concrete technology and its diverse uses are by no means totally new. In Norway, the first applications were anticipated more than 20 years ago, and microsilica concrete structures about 13 years old exist. Indeed, the basic technology derives from the extensive Norwegian experience in combination with research and development performed at various Scandinavian institutes and organizations.

This extensive research and developmerfthas not only mapped out the new property space that we can enter with microsilica concretes, but has also established remarkable links between property improvement and changes in the microstructure. It has further established the applicability of the basic principles of concrete technology to microsilica concrete, easing its specification and use.

It is important that the fundamentals of concrete technology are providing the qualitative link between properties and microstructure of these high performing concretes. Especially since these fundamentals derive from the pioneering work of T. C. Powers performed some 40-50 years ago, a comprehendible microstructural basis brings continuity to this most recent development in concrete technology and inspires additional confidence in its use.

A number of landmark projects have already been executed utilizing this relatively new technology. In general microsilica concrete is finding diverse applications ranging from roof tiles to marine and offshore structures. Its most interesting applications relate to high-performance concrete products and structures, be it in the form of normal or light weight in combination with 
high strength or high durability. The collective challenges now facing us include, first believing and understanding microsilica concretes, and second applying these products optimally.

\section{New Materials from Hydraulic Cements (Symposium L) \\ J. B. Birchall, ICI New Science Group}

Recently very significant advances have been made in the preparation of strong materials from hydraulic cements such as Portland cement or the calcium aluminate cements. Relative to common metals and plastics, the hydraulic cements are cheap in terms of energy. Portland cement, in particular, is manufactured on a huge scale $-10^{9}$ tons per year worldwidefrom readily available minerals.

When hydraulic cements are mixed with water, pastes are formed that can be easily shaped by casting or molding, and these then set or harden at ambient temperature. Everybody is familiar with the products of such technology. When cement powder and sand are mixed with water, the result is a mortar: when larger rock fragments are used in a mortar, the product is the concrete familiar in roads, buildings, and civil engineering structures. In a sense, cement paste - the product of reacting cement powder and water-is a matrix which can be filled (like a polymer matrix) with sand particles and larger "aggregates."

All these products are used in compression because cement paste and the particulate composites made from it have low tensile strength when compared to other common materials. The bend strength of a cement paste is around $10 \mathrm{MPa}$ by comparison with more than $100 \mathrm{MPa}$ for aluminum, wood, and poly(methylmethacrylate) or "plexiglas," and several hundred MPa for steel. Hence, when cement products are subject to any tensile stress, they are reinforced with steel rods or fibers of asbestos or glass. However, nature produces inorganic materials made of silica, chalk, etc., that are strong in tension (shells, for example), so why cannot man?

The ability of cement powder to form moldable pastes when mixed with water and the irreversible hardening of these pastes at ambient temperature suggests that a wide variety of products could be fabricated by low-energy processes. However, the very low tensile strength and ready fracture of the hardened material precludes such possibilities. If, for example, material of high tensile strength and enhanced fracture toughness could be made, then water would replace heat as the cause of the atomic mobility needed in sintering.

Recent work has established that the cause of the poor tensile properties of cement paste is the presence of relatively large voids of pores which result from air bubbles or poor packing of the cement grains in the original mix. Water-rich regions in the paste result in flaws in the structure of the hardened paste. Cement paste is highly porous (25-35\% volume porosity), but its strength in tension is largely determined by the larger (around $1 \mathrm{~mm}$ effective length) voids.

Methods have been developed by which large voids can be eliminated and cement grains packed tightly together so that the total porosity volume can be less than $1 \%$ by volume. Such material - which has been termed "macrodefect-free" cement-is ceramic-like in appearance and has properties quite unlike conventional cement. Typically, bend strength is in excess of $150 \mathrm{MPa}$ and fracture toughness tenfold that of a conventional paste. Polymeric rheology aids are used in processing, and the combination of this with water and cement powder, following high shear mixing, produces a plastic-like dough which can be press or injection molded or extruded (by the techniques common in the plastics industry) and the hardening of the formal material greatly accelerated by gentle $\left(<100^{\circ} \mathrm{C}\right)$ heating.

The products are exciting not simply for their good mechanical properties, as demonstrated by the manufacture of a cement spring, but because of their attractive appearance and novel properties. Certain electrical properties (such as arc resistance) have been found to be advantageous. Mechanical properties at low temperature (e.g., $-196^{\circ} \mathrm{C}$ ) are retained and this, together with low permeability to gases, suggests application in cryogenics. The materials have excellent acoustic damping characteristics and a hi-fi loud speaker made from MDF cement in on the market in the United Kingdom. The properties of MDF cement are sufficiently ceramic-like to cause damage to projectiles, suggesting its use as armor. Of particular interest in this respect is the ability to reinforce an MDF cement matrix with an organic fiber such as nylon to produce a material with high impact resistance. Such a material approaches the paradox of a nylon fiber-reinforced ceramic. Filling the paste with iron powder gives material able to screen electromagnetic radiation. Many other applications are suggested by the unique combination of properties of these new materials which are at present under widespread evaluation.

\section{Symposium M: Coal Combustion and Conversion Wastes: Characterization, Utilization and Disposal}

\section{Disposal of Fly Ash and Flue Gas Desulfurization Waste in the Northern Great Plains (Symposium M)}

G. H. Groenewold, University of North Dakota and D. J. Hassett, North Dakota State University

The use of low-sulfur, low-rank Western coals for electric power generation has dramatically increased over the last decade. Clean air legislation has mandated the reduction of particulate and sulfur dioxide emissions to the atmosphere. Particulates removed from the power plant stack emissions are referred to as fly ash. The removal of sulfur dioxide from the stack emissions results in the generation of flue gas desulfurization (FGD) waste. As a consequence, fly ash and FGD solid waste accumulate at the power plant forming a large volume of waste that must undergo disposal in an environmentally safe manner. In the Northern Great Plains most power plants dispose of fly ash and FGD waste in the adjacent surface coal mine pits. Presently, approximately $1,750,000$ tons of these wastes are generated in North Dakota on an annual basis. These quantities will increase significantly as additional thermo-electric and lignite gasification facilities are constructed.

Coal contains small amounts of toxic trace elements which are concentrated in the fly ash and FGD waste. Trace elements of particular concern in the Northern Great Plains include arsenic, selenium, and molybdenum. This has raised concerns about the potential effects of these wastes on groundwater in disposal areas.

The potential impacts of fly ash and FGD waste on ground water quality by disposal in various surface mine settings is being evaluated by the North Dakota Mining and Mineral Resources Research Institute at the Center Mine near Center, North Dakota. The research, presently funded by DOE, involves detailed field monitoring and laboratory studies and has been ongoing since 1978.

Monitoring data from the Center Mine indicate that groundwater adjacent to or below FGD waste is characterized by concentrations of sodium and sulfate that are considerably greater than the average for those ions in unaffected groundwater. The mineralization of FGD waste-affected groundwater at the Center Mine ranges from 6,564 to 9,521 parts per million. The average mineralization of unaffected groundwater at the Center Mine is 2,200 parts per million. The concentrations of arsenic and selenium in FGD waste-affected groundwater are within the same ranges as groundwater in unaffected areas (typically 10-20 parts per billion). Molybdenum concentrations show considerable variability, ranging from 70 to 51,180 parts per billion in FGD waste-affected groundwater. The most significant impacts of FGD waste on groundwater quality have occurred around disposal sites which are below the water table.

Groundwater impacted by the fly ash leachate shows highly variable concentrations of sodium and sulfate. Total mineralization of fly ash-affected groundwater ranges from 5,425 to 52,650 parts per million. Arsenic concentrations range from 1 to 613 parts per billion in fly ash-affected groundwater. Selenium ranges from 1 to 800 parts per billion. In all cases, however, the concentrations of arsenic and selenium are significantly below levels designated as hazardous by federal regulations. The fly ash-affected groundwater is also very alkaline.

The mobility of the toxic trace elements in ground water around the fly ash disposal sites is probably related to the alkalinity of the leachate. Current studies are evaluating the natural ability of disposal site soils to make the fly ash leachate less alkaline and thereby decrease the concentrations of toxic trace elements in impacted groundwater. Initial results of these studies suggest that most of the soils will rapidly decrease the alkalinity of these leachates, thereby prompting attenuation of toxic trace elements. As a result, the potential for long-term adverse impacts on groundwater due to high concentrations of toxic trace elements is significantly reduced in the disposal areas. However, no natural attenuation mechanisms have been recognized which will decrease the impacts of major chemical species, such as sodium and sulfate, on ground water in the disposal areas. 


\section{Symposium N: Scientific Basis for Nuclear Waste Management}

\section{Repository Performance of Nuclear Waste Glass (Symposium $\boldsymbol{N}$ ) M. J. Plodinec, E.I. DuPont de Nemours, Inc.}

At the Savannah River Plant, construction of what will be the world's largest solidification facility for nuclear waste has been underway for over a year. In this plant, the nearly $\mathbf{3 0}$ million gallons of high-level waste from production of nuclear materials for national defense will be converted from a liquid slurry to a durable borosilicate glass. Every day, a stainless steel canister will be filled with waste glass and welded closed in this plant, called the Defense Waste Processing Facility (DWPF).

By law, these canisters of waste glass will eventually go to a federal repository for permanent disposal. Attention is now turning to determining just how safe the DWPF glass will be in actual repository environments. Scientists at the Savannah River Laboratory (SRL) are carrying out an aggressive program to quantify how well waste glass will in the long term prevent release of long-lived radioactive species such as plutonium. They are finding that this glass will be an important barrier to the release of hazardous radionuclides to the environment, and that repositories containing the glass should be able to meet all federal regulations.

There are two majors parts of the SRL program. In the first, SRL is performing repository simulation tests in the laboratory using both actual and simulated waste glasses. In these tests, waste glass and stainless steel (simulating a breached canister) are placed in a cup made out of rock from potential repository sites - tuff from Nevada, basalt from Washington, or salt representing several possible sites, from Utah to Mississippi. Then groundwater is placed in the rock cups, and the cups are closed. For the tests in tuff, actual ground water from the Nevada test site is used. In the case of the basalt, a special groundwater recipe is carefully prepared in an air-free environment. For the salt tests, brines which are more corrosive than seawater are used.

Although the laboratory tests have not yet been completed, they all indicate that the amount of radioactivity which will be free to travel with the ground water will be as little as one billionth of the amount in the waste glass. These results are also being confirmed in another part of SRL's program, in which samples of simulated waste glass are buried deep underground. The most extensive tests to date have been carried out in the Stripa mine in Sweden, where samples of several simulated waste glasses have been buried in granite for over a year. In this joint effort, scientists from SRL, KBS (the Swedish nuclear program), and the University of Florida are finding that there was only a slight interaction between glass and ground water in the first month of testing; thereafter, there was almost none. This is direct support for the laboratory tests which also find that a steady state is acheived rather quickly.

A more extensive sets of burial tests has just begun in the salt test facility at the WIPP site in New Mexico. In these tests, samples of simulated waste glasses from seven countries are being emplaced in salt approximately 2,000 feet underground. These samples will be subjected to brine attack under both expected conditions, and under unexpected but possible conditions in a salt repository. In a later series of tests, full-scale canisters of glass from the DWPF will be placed in the WIPP salt and their performance tested in a variety of ways. These tests are scheduled to begin in approximately 1990, and will demonstrate both glass durability and the retrievability of canisters from a salt repository.

\section{WIPP Waste Package Testing on Simulated DHLW: Emplacement (Symposium N)}

\section{Martin A. Molecke, Sandia National Laboratories}

Several series of simulated (nonradioactive) defense high-level waste (DHLW) underground experiments have recently been installed in the Waste Isolation Pilot Plant (WIPP). The WIPP is a Department of Energy research and development facility in bedded rocksalt, 2,1 50 feet deep, located 35 miles east of Carlsbad, New Mexico. The WIPP facility has been authorized to demonstrate the safe disposal of radioactive wastes resulting from the defense activities and programs of the United States and is exempted from regulation by the Nuclear Regulatory Commission. The experiments are being conducted and closely monitored by Sandia National Laboratories, Albuquerque, New Mexico.
The primary purpose of these three to seven year-long experiments is to evaluate the underground durability of high-level waste package materials, or engineered barriers, including the waste canisters, secondary canisteroverpacks, backfilling materials (a clay-sand mixture, or crushed rocksalt), and the waste form itself, a type of high-integrity, borosilicate glass. These experiments in the WIPP include 18 full-size DHLW canisters about $2 \mathrm{ft}$ wide $x 10 \mathrm{ft}$ tall, inserted into vertical boreholes in the floor of the salt facility. The canisters are fabricated out of either a special titanium alloy or thick steel.

Most of these DHLW test packages contain electric heaters, to stimulate the heat generated by actual radioactive wastes. Several contain nonradioactive DHLW glass from a prototype facility at the Savannah River Laboratory.

\section{Symposium O: Alloy Phase Diagrams}

\section{Interfacial Phase Diagrams and Equilibrium Crystal Shapes (Symposium O) \\ Michael Wortis, University of Illinois}

The equilibrium shapes of large single crystals of pure solid materials are symmetrical and often attractive. At low enough temperatures, they consist mainly of a set of atomically flat, gemlike facets, which meet at sharp edges. It is believed that at absolute zero the equilibrium crystal surface is composed entirely of such facets.

The work reported in this paper is a theoretical investigation of the characteristic ways in which equilibrium crystal shapes evolve with increasing temperature. This evolution depends in detail on the particular material being considered; nevertheless, generic behavior can be described. Smoothly rounded regions first appear at the edges or corners of the fully faceted crystal. With increasing temperature, the faceted regions shrink in size relative to the smoothly rounded regions. Eventually, each type of facet disappears (i.e., its size shrinks to zero) at a characteristic "faceting temperature" (or "roughening temperature"), above which that region of the crystal surface is smoothly curved everywhere. High-symmetry facets (i.e., facets with simple orientations relative to the axes of the bulk crystal) disappear at higher temperatures than low-symmetry facets. If the crystal has not melted first, its entire surface is smoothly curved above the last faceting temperature.

This paper focuses on recent advances in the understanding of certain special details of this process. One example is the shape of the region near the "edge" between a flat facet and an adjacent smoothly rounded region. Such an edge can be "sharp" (i.e., with a discontinuity of slope between the facet and the rounded region) or "smooth" (no slope discontinuity). In the latter case, the way the rounded region curves away from the plane of the facet in now believed to be described mathematically by a power law $y=x \theta$. The power $\theta$ is called a "critical exponent" and its predicted value $\theta=3 / 2$ contrasts with the naively "classical" behavior $\theta=2$. Another example is the precise manner in which the area of a facet shrinks to zero as the temperature $\mathrm{T}$ is raised towards the appropriate facetting temperature $T_{R}$. Here, the "classical" expectation is that the area should approach zero proportionally to the temperature difference $\left(T_{R}-T\right)$. By contrast, the prediction of the modern theory is that the area shrinks faster than any power of $\left(T_{R^{-}} T\right)$.

These new predictions have received some limited confirmation from recent experiments carried out by groups in France, Israel, Japan, and the Soviet Union. The experiments study small metal crystals (lead and gold) about one thousandth of an inch in diameter at high temperatures and larger (quarter-inch) crystals of helium at temperatures only one degree above absolute zero.

It is not easy to perform experiments which reliably measure equilibrium crystal shapes, especially at low temperatures. The reason is that, in order to establish the true equilibrium shape (as opposed to a non-equilibrium "growth form"), atoms must be able to move from one place to another over the crystal surface. This motion is typically very slow at low temperatures, so that very long times are required to assure equilibrium. Helium has, at extremely low temperatures, a special property, called "superfluidity," which makes it an exception to this rule. 


\section{N. J. Saunders and A. P. Miodownik, University of Surrey}

Alloys of different metals contain a variety of internal structures whose origin scientists have tried to explain for over a century. These structures are not an intrinsic property of the material but also depend on external circumstances such as temperature and pressure. This is analogous to the behavior of water, which is well known to turn to ice or steam as the temperature or pressure is changed. Changes in structure, such as melting, are accompanied by very marked changes in properties, making it important to be able to define the equivalent critical conditions in alloys accurately.

Here the changes are more complicated because there are many more possibilities of structural change when the number of alloying additions is increased. Since the performance of any product ultimately rests on the stability of its components, and this holds right down to the various components inside the materials themselves, there is considerable interest in knowing when changes are likely to occur.

What is required, therefore, is a map which accurately indicates the limiting conditions under which any particular crystal structure or mixture of structures is to be found. Such maps are called phase diagrams, each phase being associated with a particular arrangement of the atoms in space. In the early part of the century such phase diagrams had to be determined experimentally, but by the 1930 s some theoretical predictions were beginning to be made concerning the regular appearance of particular crystal structures in different alloy systems. These predictions were based on a consideration of the average ratio of electrons to atoms, making simplifying assumptions about which electrons should be included in the calculation. This electron theory became extremely popular, particularly for alloys of copper, silver, and gold; so popular, in fact, that leading exponents had to admit that its predictive capacity was being overrated. This was particularly so in relation to the more complicated alloys being developed for jet engine and space projects in the 1950s with the result that alternative predictive schemes began to be considered.
These alternative schemes involve taking into account many other factors besides the electronic properties such as the mechanical strain arising between atoms of different sizes and, more particularly, the thermal properties of the system. Such a thermodynamic approach is of necessity more general. Its one great advantage is that it can be formally extended to cope with many different alloying additions at the same time and lends itself more easily to computer calculations. The computerized calculation of phase diagrams known as the CALPHAD approach was initially formulated at ManLabs in Boston.

It would, of course, be extremely useful if all the earlier work could be included in the general thermodynamic framework, but it turns out that the two methods have never been compared properly on the same systems. The work presented by the University of Surrey at the 1984 MRS Fall Meeting is an attempt to perform a CALPHAD analysis for alloys of copper-zinc, copper-tin, and copper-aluminum. (The first two systems include many brasses and bronzes familiar in everyday life, but capable of improvement.)

Because the CALPHAD technique is a more general approach, some sacrifices have to be made in considering the finer detail of electronic contributions, but the results seem to indicate that the older approach based on electron theory can be encompassed within a more general thermodynamic method. This will now make it possible to go ahead with predictions on copper-based alloy systems containing complicated additions of many elements.

In common with other a reas of scientific progress, the older electron theory has not been discarded but now forms part of a more comprehensive analysis of this particular scientific field. No doubt the present thermodynamic approach can be further improved by other developments in due course; it will be interesting to see to what extent still newer electronic treatments based on wave mechanics can improve on the present state of the art.

See inside back cover for information on 1984 Fall Meeting Proceedings.

\title{
Focused lon Sources with Lenses for OEM and R\&D
}

\section{Standard systems:}

\author{
DP10-0300: Air-cooled 10mA Duoplasmatron ion source DP10" with \\ or w/o ExB unit and lenses for focused beams to $30 \mathrm{keV}$.
}

DPQ-1503: DP10 ion source and DPQ lenses for beams to $15 \mathrm{keV}$ and $1 \mathrm{~mA}$.

DPQ-2000: DP10 ion source with 2-lens column for high current beams with spot sizes from 200 to 5 microns at $5 \mathrm{~cm}$ min working distance, offering a broad range of focusing conditions on target.

\section{Optional Accessories:} ExB unit DPQ-522 with stigmatic focusing over the range, microsecond pulser DPQ-533 with sharp rise and fall times, $X, Y$ raster scanner $R S 1200$ with bipolar amplifier RS1224-600, refocus lens DPQ-536.

Used for surface analysis, surface modification studies, with lasers for resonance ionization spectroscopy, in the terminal of higher energy accelerators used for tribology, ion implantation, solid state physics, neutron production, as a terminal ion source in tandem accelerators. 